\title{
Essentially Compressible Modules Relative to A Submodule
}

\author{
Shukur Neamah Al-Aeashi ${ }^{1 *}$, Fatimah Hussein Al-Bakaa ${ }^{2}$ \\ ${ }^{1}$ Department of Mathematics, College of Education, University of Kufa \\ Najaf, Iraq \\ *Corresponding author's email: shukur.mobred [AT] uokufa.edu.iq \\ ${ }^{2}$ Department of Mathematics, College of Computer Science And Mathematics, University of Kufa \\ Najaf, Iraq \\ Email: fatimah.albakaa [AT] student.uokufa.edu.iq
}

\begin{abstract}
ABSTARCT---- In this work, the new notion is defined namely essentially compressible relative to a submodule, as a new generalization of compressible module relative to a submodule where a module $\mathcal{M}$ is called compressible module relative to a submodule $N$ of $M$ if for all non-zero submodule $\mathrm{K}$ of $\mathcal{M}$ such that contains $N$, there exists a monomorphism $f \in H o m(\mathcal{M}, \mathrm{K})$. We study some basic properties of this class and many relationships between these classes and other related concepts are presented and studied.
\end{abstract}

Keywords-Compressible module relative to a submodule, Retractable module relative to a submodule, Essentially Compressible module relative to a submodule, Essentially Retractable module relative to a submodule.

\section{INTRODUCTION}

Throughout, $\mathrm{R}$ be a ring with unity and each module be a unitary right $\mathrm{R}$-module. The concept of compressible modules introduced in 1981 by Zelmanowitz, where a module $\mathrm{M}$ is called compressible if it can be embedded in any non-zero submodule $\mathrm{A}$ of $\mathrm{M}$. In other words $\mathrm{M}$ is compressible module if for each nonzero submodule $\mathrm{A}$ of $\mathrm{M}$, there exists a monomorphism $\mathrm{f} \in \mathrm{Hom}(\mathrm{M}, \mathrm{A})$, retractable modules introduced in 1979 Khuri, where " a module $\mathrm{M}$ is retractable if every nonzero submodule A of $\mathrm{M}$, $\operatorname{Hom}(\mathrm{M}, \mathrm{A}) \neq 0$ "[16]. Moreover generalizations of these classes have been studied by several authors see [5], [7], [9], [11] and [17] a dual of retractable concept in 2006, a coretractable module appeared in [8]. However Amini [6], studied this class of modules, where " $\mathcal{M}$ is called coretractable if for all proper submodule $\mathrm{A}$ of $\mathcal{M}$, there exists $0 \neq \mathrm{f} \in_{\mathrm{Hom}}(\mathcal{M} / \mathrm{A}, \mathcal{M})$ " and then more authors studied this concept and its generalizations for more see [4], [12], [13] and [14]. "Al-Aeashi and Al-Bakaa introduce the concept of retractable module relative to a submodule where for every proper submodule $\mathrm{K}$ of $\mathrm{M}$ containing $\mathrm{N}$, there is a nonzero homomorphism $\mathrm{f}: \mathrm{M} \rightarrow \mathrm{K}$ " see [2], and then in [3], they introduce the concept of compressible module relative to a submodule where for every proper submodule $K$ of $M$ containing $N$, there is a monomorphism $f: M \rightarrow K "$ ". In this paper, we introduce a new notion of compressible module relative to a submodule. Where an R-module $\mathrm{M}$ is called essentially compressible module relative to a submodule $\mathrm{N}$ of $\mathrm{M}$ ( essentially $\mathrm{N}$-compressible) if for all submodule $\mathrm{K}$ of $\mathrm{M}$ where $\mathrm{N} \leq \mathrm{K}$ there exists a monomorphism $\mathrm{f}: \mathrm{M} \rightarrow \mathrm{K}$ such that $\mathrm{f}(\mathrm{M}) \leq_{\mathrm{e}} \mathrm{M}$. Some basic properties of these notions are given in addition we introduce the concept of small kernel compressible module where an R-module $\mathrm{M}$ is called small kernel compressible module ( briefly, sk- compressible module ) if for each non-zero submodule $\mathrm{N}$ of $\mathrm{M}$, there exists $\mathrm{f}: \mathrm{M} \rightarrow \mathrm{N}$ such that $\operatorname{kerf} \ll \mathrm{M}$, we shall denote $\leq,<, \leq_{\mathrm{e}}$ and $\operatorname{End}(\mathrm{M})$ for the submodule, small submodule, essential submodule and the endomorphism of a module M respectively.

\section{PRELIMINARIES}

Definition (2.1): An R-module $\mathrm{M}$ is called retractable if for all non-zero submodule $\mathrm{N}$ of $\mathrm{M}$, there exists a non-zero homomophism $\mathrm{f:M} \rightarrow \mathrm{N}$.

Definition (2.2): An R-module $\mathrm{M}$ is called compressible if for all non-zero submodule $\mathrm{N}$ of $\mathrm{M}$, there exists a non-zero monomorphism $\mathrm{f}: \mathrm{M} \rightarrow \mathrm{N}$.

It is clear every compressible module is retractable. There are some generalizations of these concepts as follows:

Definition (2.3): An R-module $M$ is called small compressible if $M$ can be embedded in each of its non-zero small submodule.

Equivalently, $\mathrm{M}$ is small compressible if there exists a monomorphism from $\mathrm{M}$ into $\mathrm{N}$ whenever $0 \neq \mathrm{N} \ll \mathrm{M}$. Where a submodule $\mathrm{K}$ of $\mathrm{M}$ is called small in $\mathrm{M}(\mathrm{K}<<\mathrm{M})$, in case $\mathrm{M}=\mathrm{K}+\mathrm{L}$ implies that $\mathrm{L}=\mathrm{M}$ for any submodule $\mathrm{L}$ of $\mathrm{M}$ [17]. 
Definition (2.5): Let M be an R-module, $\mathrm{N}$ be a submodule of M. M is called retractable module relative to N ( briefly, $\mathrm{N}$-retractable) if for all non-zero submodule $\mathrm{K}$ of $\mathrm{M}$ where $\mathrm{N} \leq \mathrm{K}$ there exists a non-zero homomorphism $f: M \rightarrow K$. A ring $R$ is called retractable module relative to an ideal $I$ of $R$, if $R_{R}$ is retractable $R$-module relative $I_{R}$ [2].

Examples and Remarks(2.6) [2]:

1) Let $\mathrm{M}$ be an R-module and $N \leq M$. Then clear that every retractable module is retractable module relative to each it's submodule $N \leq M$, but the converse is not true in general, for example the module $\mathrm{Q}$ as Z-module is retractable module relative to itself but not retractable module since $Z \leq Q \operatorname{Hom}(\mathrm{Q}, \mathrm{Z})=0$.

2) The $Z$-module $Z_{4}$ is retractable module and hence is $N$-retractable module by Part(1).

3) Every compressible module is retractable relative for each submodule of it. That is clear since each compressible module is retractable module.

4) For each non-zero $\mathrm{M}$. We have $\mathrm{M}$ is M-retractable module.

5) Every semisimple R-module is N-retractable module, for all submodule $\mathrm{N}$ of M. But not conversely such as Part(2), where $\mathrm{Z}_{4}$ is $\mathrm{N}$-retractable module but not semisimple.

Definition(2.7): Let $\mathrm{M}$ be an R-module, $\mathrm{N}$ be a submodule of $\mathrm{M} . \mathrm{M}$ is called compressible relative to $\mathrm{N}$ (briefly, $\mathrm{N}$ compressible) if for all non-zero submodule $\mathrm{K}$ of $\mathrm{M}$ where $\mathrm{N} \leq \mathrm{K}$, there exists a monomorphism $f: M \rightarrow K$. A ring $\mathrm{R}$ is called compressible relative to an ideal I of $\mathrm{R}$ if $\mathrm{R}$ is compressible $\mathrm{R}$-module relative to I[3].

Examples and Remarks(2.8) [3]:

1) Every compressible module is compressible relative to each submodule.

The converse is not true in general, for example, consider $M=Z \oplus Z$ as Z-module. $\mathrm{M}$ is not compressible module [17]. But it is N-compressible where $N=2 Z \oplus Z$ since the only submodule contains $\mathrm{N}$ are $\mathrm{M}$ and $\mathrm{N}$. So there exists identity map $f: M \rightarrow M$ where $\operatorname{kerf}=0$ and $g(\mathrm{x}, \mathrm{y})=(2 \mathrm{x}, \mathrm{y})$, so $\mathrm{kerg}=0$. Thus $\mathrm{M}$ is N-compressible module.

2) Every $\mathrm{N}$-compressible module is $\mathrm{N}$-retractable module for any $\mathrm{N} \leq \mathrm{M}$.

The converse of above proposition is not true in general for example $\mathrm{Z}_{6}$ as $\mathrm{Z}$-module is $\mathrm{N}$-retractable module but not $\mathrm{N}$ compressible module for each submodule $\mathrm{N}$ of $\mathrm{Z}_{6}$.

3) A module $\mathrm{M}$ is compressible if and only if $\mathrm{M}$ is (0)-compressible.

\section{THE MAIN RESULTS}

In this section, we shall introduce a new generalizations of the notation compressible module namely small kernel compressible module where we find each compressible module is small kernel compressible module and we introduce the notion of essentially compressible relative to a submodule as a new generalization of compressible module relative to a submodule.

\section{Definition(3.1):}

An R-module M is called small kernel compressible (briefly, sk-compressible) if for all nonzero submodule $\mathrm{N}$ of $\mathrm{M}$, there exists $f: M \rightarrow N$, $\operatorname{kerf} \ll \mathrm{M}$.

Examples and Remarks(3.2)

1) Every compressible module is sk-compressible module.

Proof:

Let $0 \neq N \leq M$. Since $\mathrm{M}$ is compressible module, so there exists $f: M \rightarrow N$ and $\mathrm{f}$ is monomorphism hence kerf $=0 \ll M$. Then $\mathrm{M}$ is sk-compressible module.

2) The converse of Part(1) is not true, for example, consider $Z_{4}$ as $Z$-module is sk-compressible module but not compressible module.

3) There is no relation between sk-compressible module and N-compressible module for each $N \leq M$.

For example, consider the example $\mathrm{Z}_{4}$ as $\mathrm{Z}$-module is sk-compressible module but not $\mathrm{N}$-compressible where $\mathrm{N}=(2)$. While see example in part(1) examples and remarks(3.2) $M=Z \oplus Z$ as Z-module is N-compressible but not skcompressible since if $N=Z \oplus(0)$, then $f: Z \oplus Z \rightarrow Z \oplus 0$ kerf $\leq Z \oplus Z$ and $\operatorname{kerf} \star \star \star * Z \oplus Z$, since the only small in $Z \oplus Z$ is $\{(0,0)\}$. 
Recall that" A submodule $N$ of an R-module $M$ is invariant if $f(N) \subseteq N$ for each $f \in E n d(M)$.Some anthers called an invariant submodule, fully invariant submodule" and " The right R-module $\mathrm{M}$ is called a duo module provided every submodule of $\mathrm{M}$ is fully invariant "[1].

\section{Proposition(3.3):}

Let $\mathrm{M}$ be a duo R-module and $\mathrm{N}$ be a submodule of $\mathrm{M}$. If $\mathrm{M}$ is $\mathrm{N}$-compressible module, then $\mathrm{M}$ is sk-compressible module.

\section{Proof:}

Let $\mathrm{M}$ be an $\mathrm{N}$-compressible module and $\mathrm{K}$ be a non-zero submodule. We must prove $\mathrm{M}$ is sk-compressible module.

If $\mathrm{K} \geq \mathrm{N}$ by $\mathrm{N}$-compressibility we have a monomorphism $f: M \rightarrow K$ and hence $\mathrm{kerf} \ll \mathrm{M}$, thus $\mathrm{M}$ is sk-compressible module.

If $\mathrm{K}<\mathrm{N}$, so $\mathrm{K}+\mathrm{N} \geq \mathrm{N}$ and again by $\mathrm{N}$-compressible module we have $f: M \rightarrow K+N$ and $i: N+K \rightarrow M$. So to $f \in E n d(M)$. But $M$ be duo module thus iof $(K) \subseteq K$ and $\operatorname{ker}($ io $f) \ll M$ since iof $f$ is monomorphism, thus $M$ is sk-compressible module .

Now, we shall define another generalization notion of N-compressible module as follows:

\section{Definition(3.4):}

Let $\mathrm{M}$ be an R-module and $\mathrm{N}$ be a submodule of $\mathrm{M} . \mathrm{M}$ is called essentially compressible module relative to a submodule (briefly, essentially $\mathrm{N}$-compressible module) if for all $\mathrm{K} \geq \mathrm{N}$, there exists $f: M \rightarrow K$ is monomorphism such that $\mathrm{f}(\mathrm{M}) \leq_{\mathrm{e}} \mathrm{M}$.

\section{Proposition(3.5):}

Every essentially N-compressible module is essentially N-retractable module.

\section{Proof:}

Let $\mathrm{M}$ be an essentially $\mathrm{N}$-compressible module and $\mathrm{K} \geq \mathrm{N}$, so there exists a monomorphism $f: M \rightarrow K$ such that $\mathrm{f}(\mathrm{M}) \leq_{\mathrm{e}} \mathrm{M}$ and hence $\mathrm{M}$ is essentially $\mathrm{N}$-retractable module $\mathbf{m}$.

The converse of proposition(3.5) is not true in general, for example, consider $\mathrm{Z}_{4}$ as $\mathrm{Z}$-module is essentially $\mathrm{N}$ retractable module but not essentially $\mathrm{N}$-compressible module.

Proposition(3.6): Let $\mathrm{M}$ be a uniform R-module and $\mathrm{N}$ be a submodule of $\mathrm{M}$, then $\mathrm{M}$ is essentially $\mathrm{N}$-compressible module if and only if $\mathrm{M}$ is $\mathrm{N}$-compressible module.

\section{Proof:}

$(\Longrightarrow)$ Let $\mathrm{M}$ be an essentially $\mathrm{N}$-compressible module and $K \geq N$, so there exists $f: M \rightarrow K$ such that $I m \leq_{\theta} M$. Therefore $\mathrm{M}$ is $\mathrm{N}$-compressible module.

( $\Longleftarrow$ ) Suppose $\mathrm{M}$ is $\mathrm{N}$-compressible module, so for each $K \geq N$, there exists a monomorphism $f: M \rightarrow K$. To prove $\operatorname{Im} f \leq_{e} M$, since $\operatorname{Imf} \leq K$ and hence $\operatorname{Imf} \leq M$, but $\mathrm{M}$ is uniform thus $\mathrm{M}$ is essentially $\mathrm{N}$-compressible module.

Proposition(3.7): Let $\mathrm{M}$ be an R-module and $\mathrm{W}, \mathrm{N}$ be two submodules of $\mathrm{M}$ such that $\mathrm{N} \leq \mathrm{W} \leq_{\mathrm{e}} \mathrm{M}$, if $\mathrm{M}$ is essentially $\mathrm{N}$ compressible module, then $\mathrm{W}$ is also essentially $\mathrm{N}$-compressible module.

Proof: Let $\mathrm{K}$ be a submodule of $\mathrm{W}$ such that $\mathrm{N} \leq \mathrm{K}$, clear that $\mathrm{K}$ is a submodule of $\mathrm{M}$ and $\mathrm{M}$ is essentially $\mathrm{N}$ compressible module, so there exists a monomorphism $f: M \rightarrow K$ such that $\mathrm{f}(\mathrm{M}) \leq_{\mathrm{e}} \mathrm{M}$, but we have $i: W \rightarrow M$ the inclusion map is also monomorphism, hence fot: $W \rightarrow K$ is monomorphism and $f \circ i(W)$ is essentially submodule of $\mathrm{M}$

Proposition(3.8): Let $\mathrm{M}$ be R-module and $\mathrm{N}_{1}, \mathrm{~N}_{2}$ be two submodules of $\mathrm{M}$. If $\mathrm{M}$ is essentially $\mathrm{N}_{1}$-compressible module and $\mathrm{M}$ is essentially $\mathrm{N}_{2}$-compressible module. Then $\mathrm{M}$ is essentially $\mathrm{N}_{1}+\mathrm{N}_{2}$-compressible module.

Proof: Let $\mathrm{N}_{1}+\mathrm{N}_{2} \leq \mathrm{K}$ so $\mathrm{N}_{1} \leq \mathrm{K}$ and $\mathrm{N}_{2} \leq \mathrm{K}$ but $\mathrm{M}$ is $\mathrm{N}_{1}$-compressible module so there exists monomorphism $f: M \rightarrow K$ and $\mathrm{f}(\mathrm{M}) \leq_{\mathrm{e}} \mathrm{M}$, likewise $\mathrm{M}$ is $\mathrm{N}_{2}$-compressible module, so $\mathrm{M}$ is essentially $\mathrm{N}_{1}+\mathrm{N}_{2}$-compressible module. 
Diagram( 3.1): Retractable And Compressible Module Relative To A Submodule

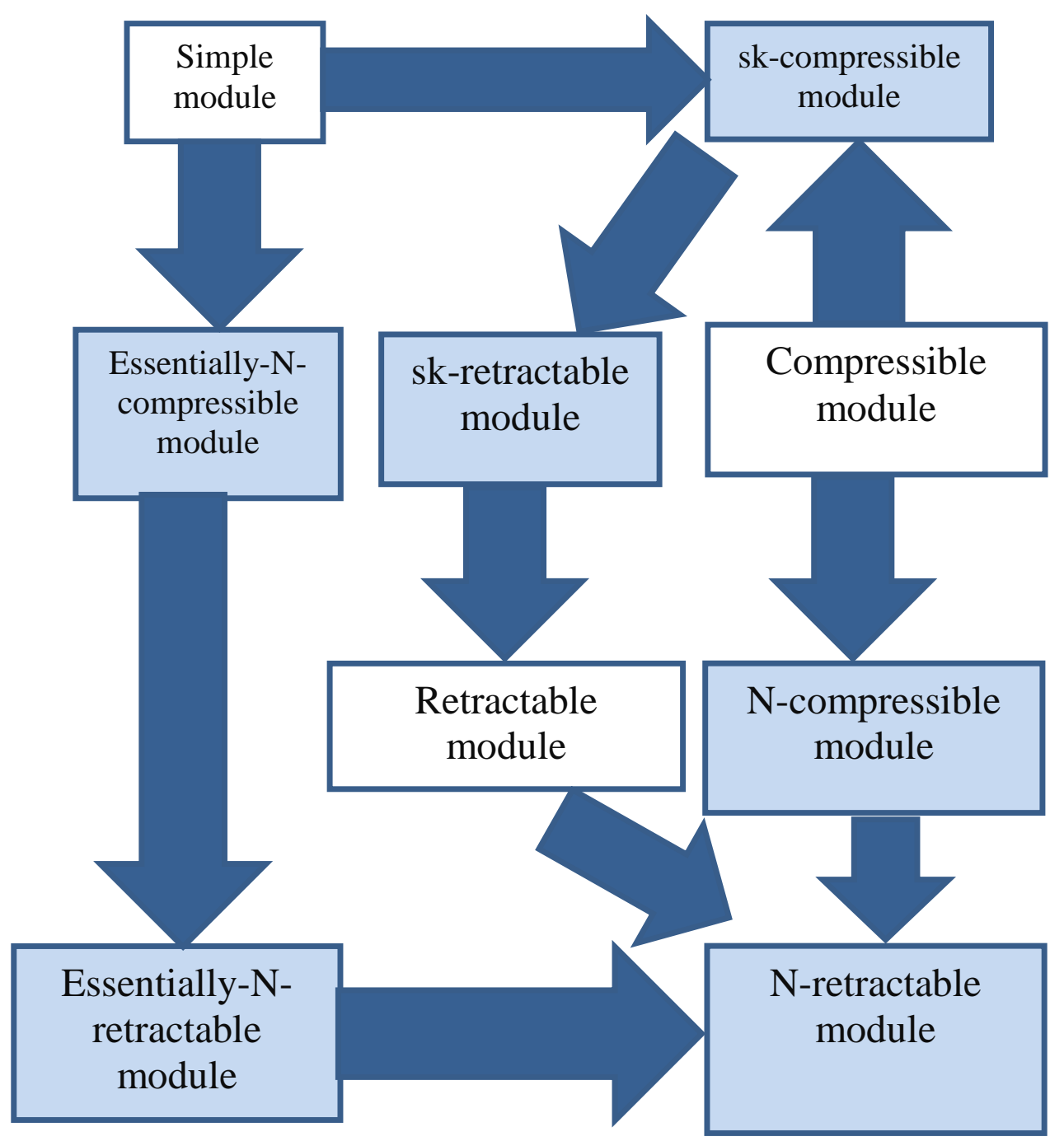




\section{REFERENCES}

[1] Abbas, M. S., On fully stable Modules, Ph. D. Thesis, University of Baghdad, 1990.

[2] Al-Aeashi S N and Al-Bakaa F. H, 2020 Retractable modules relative to a submodule and some generalizations, submitted.

[3] Al-Aeashi S N and Al-Bakaa F. H, 2020 Compressible modules relative to a submodule and some generalizations, submitted.

[4] Al-Aeashi SN, Shyaa FD, Hadi IMA. 2019 Essentially and weakly essentially coretractable modules. J. Phys.: Conf. Ser.; 1234, 012107. Doi:10.1088/1742-6596/1234/1/012107.

[5] Al-Hosainy A M A and Kadhim H 2014 A Co-compressible Module International Research Journal of Scientific Findings 1(6).

[6] Amini B, Ershad M, Sharif H. Coretractable modules. J. Aust. Math. Soc. 2009; 86(3): 289-304.

[7] Baziar M., 2009 Semi-essentially compressible modules and strongly semiprime rings, International Journal of Algebra 3 (14) 685-692.

[8] Clark J, Lomp C, Vanaja N, Wisbauer R. Lifting modules. Supplements and Projectivity in Module Theory, Frontiers in Mathematics, Birkhäuser, Basel, Boston, Berlin, 2006.

[9] Ecevit S. And Kosan M. T., 2009 "On Rings All Of Whose Modules are Retractable", Arch. Math. (Brno), 45(1), 71-74.

[10] Elman, R. Lectures on Abstract Algebra, University of California, Los Angeles, U.S.A. 1999.

[11] Ghorbani A. And Vedadi M. R., 2009 "Epi-Retractable Modules And Some Applications", Bull. Iranian Math. Soc. 35(1), 155-166.

[12] Hadi I M, Al-aeashi S N and Shyaa FD. 2019 t-Essentially and weakly t-essentially coretractable modules.

[13] Hadi I M and Al-aeashi S N 2018 Weakly coretrectable modules. J. Phys.: Conf. Ser. 1003012061.

[14] Hadi I M and Al-aeashi S N 2017 Strongly Coretrectable modules Iraqi journal of sciences 58 2C pp 1069-1075.

[15] Kasch, Friedrich. Modules and rings. Vol. 17. Academic press, 1982.

[16] Khuri S.M., The endomorphism ring of a nonsingular retractable module, East-West J. Math. 2(2) (2000) 161170.

[17] Layla S M \& Israa H M. 2016 Small Compressible Modules And Small Retractable Modules. International Journal Of Applied Mathematics \& Statistical Sciences, 5(2) 15-28.

[18] Moniri A. R, Talebi Y, 2019 Coretractable modules relative to a submodule, J. Algebra Comb. Discrete Appl. 6(2): $95-103$.

[19] Wisbauer, Robert. Foundations of module and ring theory. Routledge, 2018. 